\title{
Research on the Predicament and Countermeasures for Promotion of the Family Doctor System in Wuhou District of Chengdu
}

\author{
Wenli Deng \\ School of Public Affairs and Administration \\ University of Electronic Science and Technology of China \\ Chengdu, China
}

\begin{abstract}
This paper analyzes the current situation of the implementation of the family doctor system in Wuhou District, Chengdu, reveals the difficulties encountered in the promotion of this system, and puts forward countermeasures to promote the continuous and stable progress of it.
\end{abstract}

Keywords—family doctor system; predicament;
countermeasures

\section{INTRODUCTION}

In the past 40 years of reform and opening up, although great achievements have been made in China's economic construction, the medical level has not kept up with the pace of the times, showing an obvious backward trend. In the 19th National Congress of CPC reports, general secretary $\mathrm{Xi}$ Jinping pointed out that it is crucial to uphold and improve people's livelihood in the process of development followed by putting forward Healthy China Initiative. The implementation of family doctor system is an important part of this strategy.

In China, the grass-roots medical system led by the government, supervised by the superior health institutions and participated by the society is called the family doctor system. Through the implementation of the family doctor system, strengthening the grass-roots medical and health service system and the construction of general practitioners, it is of great practical significance to adjust the structure of China's medical system. Moreover, it helps a lot to improve the level of national health and the fairness of medical and health services.

\section{DEFINITION OF RELEVANT CONCEPTS}

\section{A. Community Sanitation Service}

Community health service is a grass-roots medical service with government as the leading, family as the unit, community residents as the object and residents' needs as the guide. It is convenient, efficient and comprehensive. At present, the service mode of "Six in One" is implemented, which is to provide services integrating prevention, medical treatment, rehabilitation, family planning guidance, health education and health care.

\section{B. Family Doctor and Family Doctor System}

According to the definition of American Academy of Family Physicians (AAFP), family doctor refers to a doctor who has received a wide range of medical professional education and training such as family health care.

Family doctor system means the system of first examine, referral and basic health care services provided by family doctors.

\section{Community First Examine and Two-Way Referral}

Community first examine refers to the service signed by community residents and their family doctors in the community hospital. In addition to emergency treatment, residents first visit the signed family doctors in the community hospital when they are ill. When the community hospital is unable to treat them, they are transferred to a specialized hospital or a comprehensive hospital for treatment.

Two-way referral refers to the process of referring to diverse medical institutions at all levels, specialized hospitals or general hospitals and community hospitals according to the severity of patients' condition and different health needs.

\section{THE CURRENT SituATION OF IMPLEMENTING FAMILY DOCTOR SYSTEM IN WUHOU DisTRICT OF CHENGDU}

Wuhou District is located in the southwest of downtown Chengdu, with Jinjiang River in the East, Shuangliu in the west, Gaoxin in the south, Qingyang in the north. It's a superior geographical location. By the end of 2017, the whole district had jurisdiction over 13 streets and 87 communities, with a permanent population of $1,319,200$, and the urbanization rate has reached $100 \%$; the whole district had more than 800 medical and health institutions at all levels, 8 first-class hospitals, 11 third-class hospitals and 8 second-class hospitals. The whole district has basically formed a benign medical structure dominated by the government, supported by institutions and participated by the society.

Wuhou District began to pilot the family doctor system in 2011. By 2017, there have been 31 community health service institutions in the District and 113 family doctor teams have 
characteristics. The pricing of service package is subject to the supervision of the District People's Congress, superior administrative departments and community residents. In this service, the personalized health management of residents is highlighted.

The "basic + personalized" signing service package proposed by Wuhou District has fully met the different needs of the residents in the area, truly focusing on the health of residents, and achieving good results in practice.

\section{DIFFICULTIES IN THE IMPLEMENTATION OF THE FAMILY DOCTOR SYSTEM IN WUHOU DISTRICT OF CHENGDU} core team, support team and auxiliary team. The core team is also the basic family doctor team configuration, which is composed of a family doctor, a medical staff and a health promotion personnel, responsible for the diagnosis and treatment of patients, referral, establishment of health records and other services; the support team is composed of TCM doctors, testers, nutritionists and other specialists in the community health service institutions; the auxiliary team is composed of community care workers and volunteers, with the supported team and auxiliary team work together to assist the core team.

\section{B. Establishing Standardized Signing Service Process}

In the process of implementing the family doctor system, Wuhou District has established a standardized signing service process.

First of all, community health service institutions and family doctor teams go deep into each community to make residents fully understand the family doctor team and contracted services.

Then, the community residents choose their own family doctors to sign the contract.

Subsequently, the family doctor team establishes health records for the contracted residents and provides various health services as agreed to perform the contracted obligations. During the whole period when the family doctor team provides services for the contracted residents, the community health institutions will continue to communicate with the residents so as to understand the service situation of the family doctor team and listen to various opinions and feedback well. Meanwhile, the residents excise supervision over the institutions in this process.

After the service expires, residents have access to choose whether to renew the contract with the original family doctor team or to re-contract with other family doctor teams.

\section{Providing "Basic Plus Personalized" Service Package}

Wuhou District actively explored the new concept of signing service package in the family doctor signing service. The content of the service package is divided into two parts of "basic + personality". The basic service content refers to 14 basic public health services provided free of charge for all contracted residents. The personalized service content is the targeted medical services provided for contracted residents in the form of private customization according to their own health

\section{A. Unreasonable Allocation of Medical Resources}

Wuhou District is the main urban area of Chengdu. West China Hospital, one of the top hospitals in China is seated here. In terms of the total amount, the medical resources in Wuhou District are significantly higher than those in other urban areas. It can not only fully meet the medical needs of this district, but also is able to undertake a large part of the medical needs of Chengdu and even Sichuan Province.

However, the allocation of medical resources in Wuhou District is not balanced. Due to the construction of major and medium-sized general hospitals in the past medical planning, most of the medical resources in Wuhou District are now in the control of a few general hospitals or specialized hospitals. In this case, the medical site, drugs and various inspection equipment of community hospitals are insufficient to meet the demand for diagnosis and treatment of common diseases, chronic diseases and frequently occurring diseases within the scope of their official duties. Therefore, they are faced with the dilemma that "there is no rice for a skillful woman"

\section{B. The Number of Family Doctors Is Short and the Level of Specialization Is Not High}

By the end of 2018, there were 1319200 permanent residents in Wuhou District. According to the principle of no more than 2000 contracted service members for per family doctor, Wuhou District needs at least 660 family doctors, while at present, there are only less than 200 ones in Wuhou District. The number of family doctors is in serious shortage.

At the same time, with the continuous improvement of living standards, residents' demand for medical and health services has changed accordingly, hoping to obtain more diversified, professional and high-quality medical services, which puts forward higher requirements for family doctors. So, on the one hand, it is necessary for family doctors to be equipped with professional general practice knowledge; on the other hand, because family doctors often go deep into the community and carry out face-to-face communication with residents, they must also master some arts of sociology, understand various customs and have communication. At present, most of the family doctors in Wuhou District are transferred from their original posts and have not received the professional training of general practitioners. Because having low education background, aging knowledge system and lack of communication skills with residents, they tend to be not fully qualified for the role of family doctors. 


\section{HOW TO IMPROVE THE FAMILY DOCTOR SYSTEM IN WUHOU DISTRICT OF CHENGDU}

In developed countries in Europe and America, family doctors enjoy a very high social status and pay and welfare. But in our country, the social status and salary and welfare of family doctors are relatively low. Presently, the community health service center in Wuhou District adopts the financial management system of separation between income and expenditure. Under this system, the family doctors, as employees of public institutions, receive fixed salaries. Because most of the family doctors in Wuhou District are not highly professionally rated, their income level is generally low. Compared with the specialized doctors in general hospitals, the family doctors have low salary, poor welfare, lack of development space, low sense of self-identity and social identity. Therefore, many family doctors in Wuhou District just drift along or having the idea of job hopping.

\section{Two-way Referral System Is Not Running Smoothly}

Two-way referral is to transfer patients between superior and lower-level hospitals, specialized hospitals and general hospitals according to needs, so as to realize the medical mode of "treating minor diseases in the community, treating serious diseases in high-level hospitals, and returning to the community after operation". However, the current referral of the community outpatient service is almost a direction to the higher level, and the referral rate of the insured from the community outpatient service to the top three hospital is higher. In addition, even patients who need rehabilitation after discharge indication has been reached are rarely transferred back to the community clinic. Instead, they are still in hospital.

On the one hand, the reason that the two-way referral system does not work well is because the current community outpatient fails to provide a complete set of rehabilitation services and health guidance for the rehabilitation patients. In turn, the rehabilitation patients have insufficient confidence in the community outpatient service; on the other hand, the major comprehensive hospitals are not willing to transfer patients based on their own economic interests.

\section{E. First Examination System of Community Outpatient Service Is Hindered}

The basis of the first examination system of community outpatient service is to realize the high proportion of reimbursement by using the medical insurance policy, and the proportion of reimbursement will be reduced or absolutely refused in case of referral or non-compliance with the regulations. However, each Social insurance participants can only select one community outpatient as a designated medical institution according to the regulations, which brings a lot of inconvenience to those insured who have different places of work and residence. At the same time, for the vulnerable groups such as the elderly and children, a single designated community clinic cannot meet their medical needs in time. Therefore, the implementation of the first examination system in community outpatient service is hindered in practice.

\section{A. Strengthening the Construction of Community Hospital and Promoting the Rational Allocation of Medical Resources}

It is necessary to reinforce the infrastructure construction of community hospitals, regularly repair them, and constantly optimize the treatment environment at the grassroots level. The corresponding diagnosis and treatment, auxiliary examination, preventive health care and health education equipment shall be provided for the community outpatient service, including oxygen supply equipment, blood glucose meter, electrocardiograph, B-ultrasound, centrifuge, blood cell counter, urine routine analyzer, biochemical analyzer, etc., so as to ensure the diagnosis and treatment ability of common and frequently occurring diseases.

It is essential to improve community outpatient drug allocation, increase input in drug varieties, and ensure that the scope, brand, and variety of drugs are gradually converged with those of large hospitals. Besides that, it is great significance of establishing the basic drug system, following the principle of classification, appropriately adjusting and expanding the types of drugs related to chronic diseases and common diseases. Then, it is wise to do a good job in docking with the medical insurance reimbursement catalog, so as to ensure the consistency and effectiveness of the drugs used by patients at the grass-roots level and in the hospital.

\section{B. Encouraging Social Forces to Provide Multi-level Signing Services}

Family doctors are featured by quasi-public products and their development cannot be separated from the guidance and support of the government. At present, Wuhou District community health service centers are all public institutions. Most of the contracted services provided by them are paid by the government, which causes the government's financial pressure to increase year by year.

To address the problem, the government should introduce relevant policies to appropriately lower the entry barrier of family doctor service providers, encourage the establishment of private family doctor teams and private community hospitals. In the meantime, it is necessary to provide multilevel contracted services for residents to choose independently. This can not only reduce the financial pressure of the government, but also offer community residents better medical and health services.

\section{Standardizing the Training Mechanism of General Practitioners and Expanding the Ranks of Family Doctors}

With the promotion of family doctors' contracted services, the demand for general practitioners has increased dramatically. In view of the current situation that the number of general practitioners is small, on the one hand, it is necessary to popularize the concept of general practice in the whole society, and carry out general practice education in the aspects of specialty setting, curriculum system construction and inhospital standardized training of general practice doctors; on 
the other hand, it is crucial to strengthen the on-the-job training and further education of the existing professionals in the community health service center. According to the actual work, the training content and direction shall be determined, excellent personnel shall be selected to study in the superior medical institutions, and relevant experts shall be invited to the community clinic for business knowledge training.

\section{Establishing and Improving the Assessment and Incentive Mechanism to Mobilize the Enthusiasm of Family Doctors}

The diagnosis and treatment services provided by family doctors are highly personalized services, which emphasize the one-to-one relationship. Therefore, it is not allowed to pay them just according to the professional title. It will be better to strengthen the performance appraisal, promote the system of "more work and more pay", which demands to include their workload, behavioral norms, technical difficulties, time for patients' disease recovery and patient satisfaction into the scope of performance appraisal. By this means, the family doctors are encouraged to advance their service quality and level. Then, they will get higher pay. Subsequently, the special promotion system of family doctors should be introduced to separate the promotion path of family doctors and specialists. In this way, it is hoped to reduce the comparative conflict between them and form an independent career development system of family doctors. This will help to enhance the professional security of family doctors.

\section{E. Improving the Two-way Referral Standard and Referral Process}

First of all, the specific disease types, and the definition basis of two-way referral should be identified, so as to ensure the upward and downward orderly flow. Only by this way, can the hierarchical diagnosis and treatment mode of "high-level hospital doing diagnosis, and grass-roots medical service institutions making implementation" be truly realized. And the technical guidance of high-level hospitals to grass-roots medical service institutions can be really implemented; secondly, it is important to build the information system, patient medical record file system, remote pathological image consultation system shared by grass-roots medical institutions and high-level hospitals, which is helpful to break the bottleneck of failure to mutual recognition of examination results. Then, the patient's health information and family genetic information can be mastered at any time. By virtue of information network, seamless connection of two-way referral through will come true.

\section{F. Carrying out Overall Planning of Community Outpatient Service in the Whole District}

The current reimbursement settlement mode is only for patients to go to a designated community outpatient, while the reimbursement proportion for non-designated community outpatient service should be reduced. In order to better meet the principle of nearby medical treatment, it is necessary to allow the same patient to designate multiple community outpatient services. For example, at both the work place and residence place, one can designate different community outpatient services based on the work certificate and residence certificate, gradually realizing the overall planning of community outpatient services in the whole district. At the same time, it is necessary to simplify the procedures for changing the designated outpatient institutions in the community, so that patients can call and log in Wechat Subscription to complete the change without leaving the home. By doing so, the community residents will truly feel that community clinic is more convenient than the major hospitals.

\section{CONCLUSION}

With the rapid development of China's social economy, people have higher and higher requirements for the quality of life and the level of medical services. Under the traditional medical system, the reality of "difficult and expensive medical treatment" has become a prominent focus of people's livelihood. The implementation of the family doctor system and hierarchical diagnosis and treatment reform will make a great difference to more reasonably allocate medical resources, promote the equalization of medical and health services, guarantee and improve people's livelihood, and heighten public health.

\section{REFERENCES}

[1] Liao Xinbo. The Dilemma of Family Doctor Signing System in China [J]. Chinese Hospital CEO, 2017 (14): 53-55. (in Chinese)

[2] Gu Xiaoli, Yin Xuan, Qian Dongfu. Analysis of Domestic and Foreign Experience and Implementation Strategies of Hierarchical Diagnosis and Treatment [J], Chinese General Practice, 2016,19 (28): 3404-3408.

[3] Xu Jie. Family Doctor: the "Gatekeeper" of Residents' Health [J]. Family Services, 2017 (4): 14-18. (in Chinese)

[4] Liu Yongjun, Liu Na, Zhang Qi. Remuneration Incentive Policies for General Practitioners in the UK and their Implications for Reference [J]. Chinese General Practice, 2018, 21 (25): 13-17 + 23. (in Chinese)

[5] Zhang Liping, Ma Xiujun, Xia Qingshi. Training Mode of General Practitioners abroad and Its Enlightenment to China [J]. Shanxi Medical Journal, 2018, v.47 (09): 52-54. (in Chinese)

[6] Xu Jing, Qian Dongfu. Discussion on the Payment Methods of General Practitioners Abroad [J]. Chinese Health Human Resources, 2015 (1): 28-30. (in Chinese)

[7] Pan Ying, Lu Yuan, Yu Dehua. The Current Situation of Multi-point Practice of General Practitioners at Home and Abroad and Its Enlightenment on the Application of Internet to Promote Hierarchical Diagnosis and Treatment [J]. Chinese General Practice, 2017 (15). (in Chinese)

[8] Guidance of the State Council on the Establishment of the General Practitioner System (GF [2011] No. 23) [DB / OL]. (in Chinese) 